\title{
Praxis matemática: reflexiones sobre la cognición que la hace posible ${ }^{1}$
}

\author{
Rafael NúÑEZ
}

Recibido: $19 / 07 / 2017$

Versión final: 28/02/2018

BIBLID 0495-4548(2017)33:2p.271-283

DOI: $10.1387 /$ theoria.17999

RESUMEN: La matemática forma un cuerpo único de conocimiento. Entre otras cosas, es abstracta, exacta, eficaz, simbolizable y proporciona sorprendentes aplicaciones al mundo real. En el campo de la filosofía de la matemática el estudio de la práctica matemática ha devenido gradualmente una importante área de investigación. ¿Qué aspectos de la mente y el cuerpo humano hacen posible la particular práctica matemática? En este artículo, reviso brevemente algunas dimensiones cognitivas que juegan un papel crucial en la creación y consolidación de la matemática.

Palabras clave: Matemática, abstracción, cognición, lenguaje, práctica, imaginación, filosofía de la matemática.

ABSTRACT: Mathematics is a unique body of knowledge. Among others, it is abstract, exact, efficient, symbolizable, and it provides astonishing applications to the real world. In the domain of philosophy of mathematics the study of the practice of mathematics has gradually become an important area of investigation. What aspects of the human body and mind make the peculiar practice of mathematics possible? In this article, I briefly review some cogntive dimensions that play a crucial role in the creation and consolidation of mathematics.

Keywords: Mathematics, abstraction, cognition, language, practice, imagination, philosophy of mathematics.

La matemática constituye un sistema conceptual extraordinario. Es abstracta, muy eficaz, precisa y objetiva. Además, genera el lenguaje fundamental de muchas disciplinas científicas, ha intrigado a filósofos a lo largo de los siglos y se encuentra en el núcleo de programas escolares de todo el mundo. Pero, ¿qué es la matemática? ¿Cuál es su naturaleza? Y, ¿de dónde procede? En el mundo académico, estas cuestiones han sido abordadas principalmente en el dominio de la filosofía y de la lógica formal, pero hoy, en los comienzos del siglo XXI, este enfoque parece demasiado restrictivo y poco útil. Defenderé aquí la idea de que estas cuestiones pueden y deben ser abordadas de manera empirica, más allá de la argumentación puramente lógica e ideológica, y especialmente a través del estudio científico de la abstracción, el lenguaje, la imaginación y las prácticas humanas. La complejidad y el aspecto multidimensional de estos dominios exigen de esta empresa que sea multidisciplinar, recogiendo los esfuerzos procedentes de muchos dominios tales como la psicología, las neurociencias, la lingüística cognitiva, la antropología, la educación y la historia de la matemática.

1 Este artículo es una versión ampliada de una conferencia originalmente pronunciada en francés; traducción de María de Paz. 


\section{La matemática, un cuerpo de conocimientos único}

Una propiedad esencial de la matemática es que está constituída, desde sus pilares más fundamentales, por abstracciones mentales idealizadas que no pueden ser percibidas directamente por los sentidos. Así, el punto euclidiano o el conjunto vacío, por ejemplo —entidades fundamentales de la geometría y de la teoría de conjuntos, respectivamente-son conceptos que no pueden ser físicamente percibidos. El punto euclidiano y el conjunto vacío, a pesar de su precisión y sus identidades claramente definidas, son entidades abstractas, idealizadas, que no existen en el mundo material y por ende no son observables en el universo físico. Sin embargo, se encuentran en los fundamentos de areas como la geometría euclidiana y de la teoría de conjuntos.

La naturaleza imaginaria de la matemática se puede apreciar más claramente aún en los conceptos que se vinculan con el infinito. Dada la naturaleza finita de nuestros cuerpos y de nuestros cerebros, nos resulta absolutamente imposible tener la experiencia del infinito en sí. No obstante, el infinito es absolutamente esencial para la matemática. Se encuentra en el centro de numerosos dominios fundamentales tales como la geometría proyectiva, el cálculo infinitesimal, la topología o la teoría de conjuntos, por citar algunos. Sin el concepto de infinito, el formidable edificio de la matemática se derrumbaría. Más aún, además de la naturaleza imaginaria, la matemática tiene características únicas. Es precisa (hasta el extremo), objetiva, rigurosa, generalizable y, por supuesto, altamente aplicable al mundo real. También es extraordinariamente estable, en el sentido de que un teorema que ha sido demostrado, permanece así para siempre. Sería ilusorio pensar en estudiar la naturaleza de la matemática sin intentar explicar estas características.

¿Cuál es, entonces, la naturaleza de la matemática? ¿Cuáles son sus condiciones de posibilidad? ¿Cuál es la clave de bóveda de este fabuloso edificio lógico tan preciso y objetivo? Estas cuestiones han sido exploradas en profundidad en el campo de la filosofía de la matemática, llegando a ser en el siglo xx el objeto de estudios específicos de los campos más técnicos de la lógica formal y de la metamatemática. Desde entonces, los fundamentos de la matemática han sido en gran medida considerados como intramatemáticos (internos a la propia matemática), como si las herramientas de la lógica formal fueran las únicas capaces de proporcionar las respuestas definitivas a las cuestiones relativas a la naturaleza de la matemática. Pero, ¿pueden los fundamentos de la matemática ser ellos mismos entidades matemáticas? ¿O se situarían los fundamentos más bien fuera de la matemática? Y, si este es el caso, ¿de dónde proceden estas entidades y qué forma tienen? En el seno de los enfoques formalistas, la búsqueda de axiomas y el estudio de sus poderes deductivos llegaron a ser los principales desafíos en las investigaciones sobre la naturaleza de la matemática.

Para intentar responder a estas cuestiones, otro enfoque (ineludible) procede del antiguo platonismo, el cual, al suponer que habría un mundo de Ideas trascendente a nuestra realidad, considera las verdades matemáticas como hechos eternos dotados de una existencia independiente de la de los humanos. Esta posición, pese al lugar destacado que ocupó en el pensamiento occidental durante siglos, no es respaldada por ningún avance científico contemporáneo y no presenta ningún punto de contacto con los trabajos empíricos actuales en materia de ideas humanas y de sistemas conceptuales (sin embargo, es paradójicamente defendida como una suerte de profesión de fe por numerosos matemáticos, físicos y filósofos). Para los intelectuales que adoptan una posición post-moderna, la cuestión de la natu- 
raleza de la matemática es más simple: la matemática, tal como el arte, la poesía, la arquitectura, la música o la moda, no es más que un «constructo social». Sin embargo, al adoptar esta perspectiva, es difícil ver lo que concede a la matemática su carácter especial. ¿Qué distingue a la matemática de la moda o de la poesía, por ejemplo? Toda propuesta de explicación de la naturaleza de la matemática debe, si pretende un nivel de precisión suficiente, dar cuenta del conjunto de características ya mencionadas y que hacen de ella una disciplina única en su especie: la precisión, la objetividad, el rigor, la posibilidad de generalización, la estabilidad y la aplicación concreta al mundo real. Este es el desafío del estudio científico de la naturaleza de la matemática: aunque las entidades matemáticas (las ideas organizadas y los conceptos estables) sean abstractas e imaginarias, se cristalizan a través de particularidades biológicas y sociales del animal humano.

Para aquellos que estudian la mente humana de manera científica, el problema de la naturaleza de la matemática es, en efecto, una verdadera aporía, cuya cuestión crucial se formula como sigue: ¿cómo puede el estudio cientifico de la mente humana dar cuenta de un dominio de ideas abstracto, ideal, objetivo, preciso, sofisticado y potente, si es imposible tener la experiencia corporal directa con las entidades que constituyen la matemática en sí? En nuestro libro Where Mathematics Comes From (Lakoff \& Núñez 2000), Lakoff y yo mismo proponemos algunas respuestas preliminares a esta cuestión. Analizaremos aquí algunos de los elementos de base de esta propuesta.

\section{Algunos mecanismos ordinarios de la cognición humana que hacen posibles las prácticas imaginativas y abstractas}

Basándonos en los descubrimientos hechos en el campo de la cognición matemática y de las neurociencias de la cognición numérica y utilizando principalmente métodos pertenecientes a la lingüística cognitiva (una sub-disciplina de las ciencias cognitivas), Lakoff y yo nos planteamos la siguiente cuestión: ¿cuáles son los mecanismos cognitivos utilizados en la elaboración de las ideas matemáticas? Y, más concretamente, ¿qué mecanismos cognitivos pueden definir la organización de las inferencias de tal forma que se puedan observar en las ideas matemáticas mismas? Avanzamos la idea de que la mayoría de las entidades técnicas abstractas de la matemática se crean por medio de mecanismos cognitivos de la vida cotidiana, los que extendiendo la organización de la experiencia corporal ordinaria posibilitan la formación de conceptos puramente imaginarios. Estos mecanismos «naturales» y ordinarios incluyen, entre otros, las metáforas conceptuales (Lakoff \& Johnson 1980; Sweetser 1990, Lakoff 1993; Núñez \& Lakoff 1998; Núñez \& Lakoff 2005), las metonimias conceptuales (Lakoff \& Johnson, 1980), los «blending» conceptuales (Fauconnier \& Turner 1998, 2002), y el movimiento ficticio (Talmy 1996). Al utilizar una técnica que hemos llamado Análisis de las Ideas Matemáticas, estudiamos en detalle numerosos conceptos matemáticos en muchos campos matemáticos, desde la teoría de conjuntos al cálculo infinitesimal, pasando por la aritmética de los números transfinitos, y mostramos cómo, por medio de estos mecanismos corporeizados en lo cotidiano, los esquemas de inferencia derivados de la experiencia corporal directa en el mundo real son extendidos de manera precisa y específica para dar lugar a una nueva organización de inferencias en dominios puramente imaginarios (para detalles, ver Lakoff \& Núñez 2000). 
Tomemos como ejemplo de manifestación de metáforas conceptuales cotidianas las dos expresiones siguientes del lenguaje corriente: «Tenemos toda una vida ante nosotros» $\mathrm{y}$ «hemos dejado atrás el largo invierno». Tomadas literalmente, estas expresiones no tienen ningún sentido. «Una vida» no puede situarse físicamente «ante» nosotros de manera medible u observable, y un «invierno» no es una cosa que se pueda situar físicamente «detrás» de nosotros. Expresiones como estas, cuyo sentido no es literal sino metafórico, pueden encontrarse en muchos ámbitos del lenguaje común: «una acogida fría», «un peso en la conciencia», «un mercado a la baja». En este sentido, la metáfora conceptual no es una simple figura de estilo, ni una herramienta de comunicación excepcional reservada al poeta y al artista. Es un mecanismo de pensamiento ordinario que, mediante una operación generalmente inconsciente y fluida, impregna prácticamente todos los aspectos del lenguaje cotidiano (y técnico) de los seres humanos, haciendo así posibles tanto la imaginación como la abstracción.

La lingüística cognitiva (y, más precisamente, la semántica cognitiva) estudia este fenómeno en detalle y muestra que el sentido de estos cientos de miles de expresiones lingüísticas puede ser modelado a partir de un número relativamente reducido de metáforas conceptuales. Estas metáforas, en tanto que proyecciones que conservan las inferencias de un dominio en otro, son mecanismos cognitivos que nos permiten proyectar la estructura inferencial surgida de un dominio fuente generalmente anclado en la experiencia concreta del mundo real (como la experiencia térmica, por ejemplo), hacia otro dominio generalmente más abstracto - el dominio objetivo (como los afectos) - . En consecuencia, siguiendo con este ejemplo, las nociones específicamente vinculadas a la experienceia térmica, como «frío» o «calor», son proyectadas, respectivamente, sobre las nociones de «falta de afecto» o de «presencia de afecto», abriendo así un modo de inferencia en el que el dominio relativamente abstracto del «afecto» es concebido y comprendido en los términos de un dominio mucho más concreto - en este caso, la experiencia térmica-. Lo crucial aquí es que la organización de las inferencias es abstracta y puramente imaginaria, y que surge de una red inferencial generada a partir de las proyecciones establecidas desde un dominio fuente a un dominio objetivo.

Podemos ilustrar el funcionamiento de estas proyecciones conceptuales con ejemplos tomados del área de la conceptualización del tiempo. En este caso, es muy habitual que acontecimientos que ocurren en la esfera temporal sean concebidos metafóricamente como estando delante o detrás de nosotros, según si estos no han ocurrido aún («futuro»), o si ya ocurrieron («pasado»), respectivamente. La estructura de las inferencias de esta proyección da cuenta de un gran número de expresiones lingüísticas, tales como «el verano aún está lejos», o «el fin del mundo se acerca» y «la primavera ha llegado». Muchas de las implicaciones $-\mathrm{o}$ «verdades»- importantes se desprenden de ese acto de proyección. Por ejemplo, las propiedades transitivas que se aplican a las relaciones entre el observador (p.e., el sujeto hablante) y los objetos del dominio fuente son conservados en el dominio objetivo del tiempo: si, con respecto a la parte delantera del sujeto, el objeto A está más lejos que el objeto $\mathrm{B}$ y el objeto $\mathrm{B}$ está más lejos que el objeto $\mathrm{C}$, entonces el objeto $\mathrm{C}$ está más próximo del sujeto que el objeto A. En el dominio temporal, a través de la proyección esto significa que el instante $\mathrm{C}$ está en un futuro «más próximo» que el instante $\mathrm{A}$. Relaciones similares se aplican a los objetos situados detrás del observador y los tiempos pasados. Además, a través de la proyección metafórica, el tiempo es percibido como teniendo una extensión medible; puede ser prolongado como un segmento de un camino, concebido como un dominio 
linear delimitado, etc. Es de suma importancia aquí, notar que ninguna de estas propiedades existe en un mundo real físico, sino que son creadas a través de mecanismos de la imginación humana-en este caso la metáfora conceptual. En la sección siguiente analizaremos con más detalle aún otras propiedades de los mapeos metafóricos espacio-tiempo que nos sirven para entender la naturaleza de los conceptos matemáticos y sus bases axiomáticas.

Es importante destacar que las proyecciones conceptuales del dominio imaginario no se manifiestan solamente en el lenguaje, sino también con frecuencia en movimientos corporales específicos - en gestos espontáneos- que son co-producidos con el discurso, a menudo inconscientemente. En efecto, las investigaciones en un gran número de campos disciplinares (ya sea la psicología el desarrollo, la neuropsicología, la lingüística o la antropología) han puesto de relieve un vínculo estrecho entre la producción verbal y gestual y el pensamiento. Por ejemplo, muchos descubrimientos han mostrado que los gestos están a menudo increíblemente sincronizados con el habla (McNeill 1992), que se desarrollan en relación estricta con el habla (Bates \& Dick 2002; Godin-Meadow 2003; Iverson \& Thelen 1999), y que ciertas lesiones del cerebro que afectan al lenguaje alcanzan igualmente la producción de gestos (McNeill 1992). Además, la producción gestual que acompaña al habla es universal y se observa en todas las culturas (McNeill 1992; Kita \& Essegbey 2001; Núñez \& Sweetser 2006) es en gran medida producida inconscientemente (McNeill 1992) y puede proporcionar informaciones complementarias (o matizar) a aquellas que son transmitidas por el habla (Kendon 2000), y opera a menudo sistemáticamente en paralelo con las expresiones metafóricas lingüísticas (McNeill 1992, Núñez \& Sweetser 2006; Cienki 1998; Cornejo et al. 2009). Los resultados de estas investigaciones apoyan la hipótesis de que el lenguaje y la producción gestual son en realidad dos facetas del proceso de pensamiento. Así, el estudio de la naturaleza de la matemática, en tanto producción conceptual y producto del pensamiento, se puede beneficiar tremendamente con la investigación empírica de la cognición metafórica manifestada en acción motora a través de gestos espontáneos co-producidos con el discurso.

Con estos métodos de investigación, nos fue posible estudiar hasta qué punto las definiciones formales en matemática reflejan los significados semánticos y cognitivos de los conceptos involucrados. Por ejemplo, las nociones de límite y de continuidad de funciones parecen denotar, intuitivamente, componentes dinámicos: puntos que se desplazan, valores que se aproximan a otros, cantidades que tienden a infinito, etc. De hecho, así caracterizaron estos conceptos estudiosos como Euler, Newton, y Leibniz (en efecto, todos los matemáticos hasta los trabajos de Weierstrass y Dedekind en el siglo XIX; Núñez y Lakoff 1998; Lakoff y Núñez 2000). Por ejemplo, al caracterizar el concepto de límite de series infinitas podemos leer en el clásico texto de Courant y Robbins ¿Qué es la matemática? (Courant y Robbins 1978):

«Describimos el comportamiento de $s_{n}$ diciendo que la suma $s_{n}$ se aproxima al límite 1 cuando $n$ tiende a infinito, y que escribimos

$$
1=1 / 2+1 / 2^{2}+1 / 2^{3}+1 / 2^{4}+\ldots \gg(\text { p. 64, nuestro énfasis })
$$

Sin embargo, si consultamos textos matemáticos modernos para ver cómo se define el concepto de límite y de continuidad de funciones a menudo encontramos definiciones como la que se presenta a continuación, que no contiene ni la más mínima dosis de dinamismo. Y si 
consultamos en algún texto universitario cuál es la definición de continuidad de funciones podemos leer (Simmons 1985):

Una función $f$ es continua en un número $a$ si se satisfacen las siguientes tres condiciones:

1. $f$ es definida en un intervalo abierto que contiene a $a$

2. el límite $\lim _{x \rightarrow a} f(x)$ existe, $\mathrm{y}$

3. $\lim _{x \rightarrow a} f(x)=a$

donde la expresión $\lim _{x \rightarrow a} f(x)$ es definida como:

Sea una función $f$ definida en un intervalo abierto que contiene a $a$, posiblemente excluyendo a a propiamente tal, y sea $L$ un número real. La expresión

$$
\lim _{x \rightarrow a} f(x)=L \text { significa que } \forall \varepsilon>0, \exists \delta>0 \text {, tal que, si } 0<|x-a|<\delta \text {, entonces }|f(x)-L|<\varepsilon \text {. }
$$

Desde un punto de vista lingüístico cognitivo ninguno de los conceptos involucrados en estas definiciones formales denota movimiento. Nada se mueve, nada se desplaza, nada se aproxima a nada. Estas definiciones formales solo caracterizan cantidades estáticas y utilizan verbos de existencia (no de movimiento; por ejemplo $\forall \varepsilon>0, \exists \delta>0$ ), y la satisfacción de ciertas condiciones que son establecidas en términos de diferencias artiméticas estáticas (p.e., $|f(\mathrm{x})-L|$ ) y de relaciones estáticas «menor que» (p.e., $0<|\mathrm{x}-a|<\delta$ ). Eso es todo. No hay movimiento. Sin embargo, a través del estudio empírico de metáforas conceptuales y de gestos espontáneos hemos podido comprobar que los matemáticos profesionales frecuente y espontáneamente conciben - a pesar de lo que prescriben las definiciones formales - los conceptos de límite y de continuidad de funciones en términos dinámicos. Sus movimientos manuales - muy precisos y sincronizados con el habla - demuestran que ellos, en la práctica y en su realidad psico-cognitiva, conciben estas nociones en términos dinámicos, y ello no solo en contextos de enseñanza (Núñez 2006), sino también en contextos de colaboración profesional demostrando teoremas (Marghetis y Núñez 2013). ¿Por qué es esto relevante? Porque demuestra que si queremos investigar la naturaleza de la matemática es imperioso indagar mucho más allá de las definiciones, expresiones, y simbolismos formales. Como lo muestra la práctica de la co-producción metafórica y gestual, las ideas y conceptos matemáticos son más vastos y de mayor riqueza que lo que es expresado a través del lenguaje y las definiciones formales.

\section{La imaginación y la abstracción en la producción de axiomas que crean matemática nueva}

Como mencionamos en la sección anterior, la metáfora conceptual nos ayuda a anclar y a estructurar la conceptualización de experiencias complejas, como la del tiempo, por ejemplo. El tiempo, experiencia humana fundamental que ha intrigado a los filósofos, los teólogos y los físicos durante siglos, está íntimamente presente en todas nuestras acciones. Y sin embargo, es abstracto; nuestro cerebro no tiene una zona dedicada a la experiencia temporal pura en el sentido en el que ciertas zonas están dedicadas a la estimulación visual o auditiva que nos orientan en el espacio, por ejemplo. Si bien es cierto, poseemos mecanismos biológicos básicos para procesar y discriminar duración - los que compartimos con muchos otros animales-, nociones como «presente», «pasado», y «futuro» (llamado tiempo deíctico) son el resultado de elaboraciones conceptuales abstractas y complejas (por ejem- 
plo, ¿cómo podríamos responder en concreto, y con certeza, a la pregunta de cuál es la duración física del «presente» cuando decimos «Ahora que está usted leyendo este texto», «Ahora que termina el invierno», o «Ahora a principios de este nuevo milenio», etc.? O si prefiere, ¿cuál es la duración física que separa al «pasado» del «futuro»?; ver, Núñez \& Cooperrider 2013). Con el objetivo de conceptualizar este tipo de fenómenos abstractos, nos servimos de lo imaginario: tratamos el «tiempo» metafóricamente como si fuera de naturaleza espacial y una forma muy común de esta metáfora (especialmente en el caso del tiempo deíctico, con sus categorías «pasado», «presente», y «futuro») consiste en concebir el futuro y el pasado como estando respectivamente delante y detrás de nosotros. Esta manera - generalmente inconsciente- de pensar nos parece evidente y completamente natural, hasta el punto de que apenas observamos que se trata de una forma fundamental de comprensión de la experiencia temporal, compartida por numerosas culturas alrededor del mundo.

Sin embargo, aunque compartida por un gran número de culturas sin ninguna relación entre ellas, esta manera de concebir el futuro y el pasado no es universal. Llegamos a esta conclusión, en colaboración con la lingüista Eve Sweetser de la Universidad de California en Berkeley, estudiando en detalle las concepciones del tiempo del pueblo Aimara, en los Andes (Núñez \& Sweetser 2006). Se trata de la primera refutación registrada del supuesto (antes tomado por válido) de que la orientación metafórica del tiempo-con el futuro delante nuestro y el pasado detrás-es el resultado de fenómenos biológicos básicos que compartimos en tanto individuos de la especie humana (p.e., peculiaridades de la organización de la corteza cerebral) y que por ende son universales. Más allá de ser un dato antropológico interesante o una curiosidad etnográfica esta documentación tiene también consecuencias en el estudio de los axiomas, de la consistencia, de las inferencias y de lo que llamamos «verdad» en otros sistemas abstractos y conceptuales, tales como la matemática.

El aimara, una lengua amerindia hablada por casi dos millones de personas en el altiplano andino del oeste de Bolivia, del sudeste de Perú y del norte de Chile, presenta un contraste fascinante con las proyecciones espacio-temporales conocidas; desafía claramente los universales interculturales de la cognición metafórica. En aimara, la palabra de base para «delante» (nayra: «ojo/delante/vista») es también la expresión de base para designar el pasado, mientras que el término básico para designar «detrás» (qhipa, «espalda/detrás») es la expresión de base para hablar del «futuro». Por ejemplo, nayra mara, que se traduce literalmente por «el año ojo/delante/» significa «el año pasado», y qhipa pacha —《el tiempo detrás»- designa el futuro. Muchas otras expresiones aimara siguen este esquema, lo que nos llevó a la hipótesis intrigante de que los Aimara concebirían el futuro como estando detrás (¡y no delante!) de ellos, y el pasado estando delante (iy no detrás!) de ellos. Pero, más allá de la etimología y de las palabras utilizadas, estábamos interesados en la cuestión de saber si el pensamiento metafórico aimara (no solo los lexemas, o palabras) adopta realmente este modo. Dicho de otra forma, queríamos tener la certeza de que las expresiones no eran puras palabras muertas y fosilizadas, sin sentido metafórico inherente. Con el fin de verificar empíricamente esta hipótesis, recurrimos al estudio de los gestos mencionados anteriormente. Así, analizamos los gestos producidos por los Aimara cuando se pronuncian sobre expresiones temporales cotidianas que implican el pasado, el futuro, el instante presente, etc. Colaborando con nuestros colegas chilenos Manuel Mamani y Vicente Neumann, de la Universidad de Tarapacá, y Carlos Cornejo de la Pontificia Universidad Católica de Chile, llevé a cabo entrevistas etnográficas filmadas con los Aimara del extremo 
nordeste de Chile, en la cordillera de los Andes, junto a la frontera boliviana. Así, pudimos observar que los hablantes Aimara producen gestos en el sentido opuesto de hablantes occidentales que se han estudiado (p.e., hablantes de lenguas indo-europeas como el inglés, el castellano, el francés). Además del campo léxico del espacio que se utiliza para representar el tiempo como estando delante (nayra) y detrás (qhipa) de ellos, representan gestualmente el tiempo como un espacio centrado de manera deíctica: el espacio del interlocutor significa esencialmente «ahora», como en el gesto de una persona occidental. El espacio detrás del interlocutor corresponde al futuro, mientras que el espacio delate del interlocutor corresponde al pasado.

Más aún, un análisis detallado de los gestos nos permitió concluir que los lugares situados delante del hablante y más próximos de él/ella corresponden a épocas recientes, mientras que los lugares situados lejos delante de él/ella corresponden a épocas más antiguas. Por ejemplo, un hablante hacía la distinción entre «el año pasado» y «este año» apuntando primero a un lugar distante al frente de él y después a un lugar próximo junto a sus pies.

Además de su valor anecdótico, estas observaciones son cruciales, en el sentido de que muestran que los significados subyacentes, y que constituyen a la abstracción humana incluso en sus niveles más fundamentales, no están determinados por fenómenos puramente biológicos. Muestra que no hay un modelo único y universal del modo de abstracción, incluso en el caso de una entidad tan fundamental como el tiempo. La biología humana es ciertamente una base fundamental de la imaginación humana. Sin embargo, partiendo de características anatómicas y de organizaciones neuronales universales en el seno de una especie, diferentes aspectos de la experiencia corporal son susceptibles de ser movilizados para la construcción sistemática de conceptos más abstractos, lo cual abre el campo a una cierta plasticidad y a variaciones culturales. Así, en lo que concierne al uso metafórico temporal de las relaciones adelante-atrás, nosotros, los occidentales hablantes de lenguas indo-europeas, por ejemplo, parecemos dar prioridad a la experiencia dinámica del desplazamiento frontal. Partiendo de este postulado de base (o «axioma») que se funda-en su forma más prototípica-sobre el movimiento hacia adelante, si andamos (hacia delante), vamos necesariamente a alcanzar un lugar situado delante de nosotros dejando detrás nuestro punto de partida. Este lugar es alcanzado en el futuro con respecto al instante de nuestra partida y el lugar de partida de donde salimos (en el pasado) está situado detrás de nosotros. Aunque los Aimara marchen en el mismo sentido que todo el mundo, ellos se basan, por su lado, en un postulado (o «axioma») radicalmente diferente. Revelan aspectos completamente diferentes de las características del delante y del atrás: lo que es percibido visualmente (y por tanto conocido) está delante del observador y lo que está detrás de él/ella está fuera del campo visual y es, por tanto, inobservable y desconocido. Estas características corresponden a propiedades esenciales de la experiencia temporal: los acontecimientos del pasado son conocidos, mientras que los del futuro no lo son. En Aimara, la percepción visual parece jugar un papel preponderante en la construcción de los conceptos relativos al tiempo, lo que es corroborado por muchas fuentes de información, desde el estudio de marcadores gramaticales de evidencia al de las prácticas y valores en la sociedad aimara (Núñez \& Cornejo 2012).

De este análisis nos quedamos con que los seres humanos disponen al menos de dos maneras de concebir el tiempo deíctico (pasado, presente, futuro) a lo largo de un eje corporal que va de adelante hacia atrás (sagital), cada una dotada de una coherencia interna, pero las dos no presentan coherencia mutua. Estas formas son definidas por las maneras mutuamente exclusivas de orientar el cuerpo en el espacio sagital unidimensional, que compo- 
nen redes inferenciales radicalmente diferentes. Sustentados por diferentes aspectos de la experiencia corporal, se obtiene, en un caso, un sistema fundamentado sobre un postulado («axioma») que sitúa al observador «frente» al futuro y otro caso, que partiendo de un postulado inverso, sitúa al observador «frente» al pasado. Una vez definidas estas orientaciones, se desprenden de ellas una serie de «teoremas» sobre relaciones temporales (p.e., las relaciones transitivas mencionadas anteriormente). Si nos preguntáramos, entonces, ¿dónde se sitúa «verdaderamente» el pasado? ¿Delante nuestro? ¿Detrás? ¿Cuál de éstas orientaciones es, en definitiva, la «correcta» o la «verídica»? A la manera de la matemática, no hay aquí respuesta trascendental definitiva.

En efecto, en muchos dominios de la matemática — tales como la geometría y la teoría de conjuntos- hay teorías que también están dotadas de una coherencia interna y que son mutuamente inconsistentes. Este es el caso, por ejemplo de los hiperconjuntos, inventados más o menos recientemente (Barwise y Moss 1991). Los conjuntos estándares gobernados por sistemas axiomáticos como el de Zermelo-Fraenkel, mediante el «axioma de fundamento», no permiten conjuntos que sean miembros de sí mismos. Pero algunos matemáticos, viendo constreñimientos a menudo innecesarios en esto, inventaron los hiperconjuntos, que gobernados por el axioma opuesto llamado de «anti-fundamento» sí permiten a los conjuntos ser miembros de sí mismos. Desde un punto de vista de la cognición metafórica es interesante hacer notar que para justificar las explicaciones del por qué es necesario crear este nuevo tipo de conjuntos, matemáticos como Barwise y Moss (1991) se refirieron a las limitaciones de «la metáfora de la caja» («box metaphor», en sus palabras) que dificulta concebir a los conjuntos como siendo miembros de sí mismos. Lo que hicieron entonces fue cambiar el dominio origen de la proyección metafórica, y en vez de usar un dominio basado en cajas o continentes, usaron uno basado en grafos (con flechas entre puntos denotando membresía y conjuntos, respectivamente), que posibilitan sin problemas denotar y concebir la auto-membresía (con flechas que se vuelven hacia el mismo punto de origen). De esta manera, en matemática, hay sistemas axiomáticos en los que los conjuntos no pueden ser miembros de sí mismos (gobernados por el «axioma de fundamento») y otros donde sí lo pueden ser (gobernados por el «axioma de anti-fundamento»). Y estos, como en el caso de las concepciones cotidianas de tiempo deíctico, basados en metáforas conceptuales diferentes-los primeros en metáforas de contenedor y los segundos en grafos-son internamente consistentes pero mutuamente inconsistentes (para detalles, ver Lakoff y Núñez 2000).

\section{Discusión}

En lo que respecta al estudio del imaginario humano, los sistemas conceptuales que de él se desprenden y las prácticas culturales subyacentes, podemos retener tres propiedades importantes, las cuales se aplican tanto a la abstracción cotidiana como a la abstracción técnica tal y como se practica en matemática:

(1) la naturaleza corporeizada de la abstracción,

(2) la naturaleza supra-individual de los conceptos,

(3) la relatividad de la verdad en los conceptos imaginarios.

Veamos cada una de estas propiedades por separado. 


\section{LA NATURALEZA CORPOREIZADA DE LA ABSTRACCIÓN}

El análisis de los mecanismos cognitivos que sirven para dar sentido a la experiencia temporal nos ha revelado que si podemos desarrollar sistemas conceptuales abstractos es porque somos seres biológicos dotados de características morfológicas y anatómicas. En este sentido, la abstracción humana es corporeizada por naturaleza. Por ejemplo, es porque somos seres vivientes dotados de un lado delantero y de un lado trasero claramente distintos, que podemos basarnos en estas propiedades y en las experiencias corporales correlativas para producir conceptos estables como el de «el futuro ante nosotros». Esto no sería posible si nuestro cuerpo fuera como el de la medusa, por ejemplo. Sin embargo, aunque otros primates comparten estos atributos morfológicos y están igualmente dotados de lados de delante y de atrás, los mecanismos cognitivos corporeizados tales como la metáfora conceptual que permiten extender sistemáticamente las experiencias corporales para crear nociones imaginarias como la de el-futuro-en-tanto-que-lugar-delante-del-sujeto, parecen ser exclusivos del homo sapiens. Lo que es más, las propiedades biológicas y las especificidades de la experiencia corporal humanas limitan fuertemente el campo de los conceptos que pueden ser creados.

Por eso los sistemas conceptuales abstractos no son «simplemente» construidos socialmente como meras convenciones. Mientras que las convenciones sociales generalmente presentan una laxitud considerable, este no es el caso con la mayoría de los conceptos abstractos humanos. Por ejemplo, el motivo de los colores de los billetes de Euro ha sido construido socialmente por convención. Y más o menos cualquier ordenación de colores habría podido servir. En el caso de las concepciones metafóricas del tiempo no es posible contentarse con cualquier dominio fuente: las concepciones humanas son espaciales. $\mathrm{Y}$ esto es un dato empirico, no una afirmación arbitraria o especulativa, puesto que no existe, que sepamos, ni lengua ni cultura que conciba el tiempo en términos térmicos o cromáticos, por ejemplo. La abstracción humana no es, por tanto, una simple «construcción puramente social». Está construida a partir de fuertes restricciones biológicas y cognitivas que no tienen nada de arbitrarias y juegan un papel esencial en la constitución de la abstracción humana, desde las ideas de lo cotidiano hasta la matemática más avanzada. La cognición y la abstracción humanas están corporeizadas, formadas por restricciones propias a la especie y no arbitrarias.

\section{LA NATURALEZA SUPRA-INDIVIDUAL DE LOS CONCEPTOS}

El estudio de la corporeización de los sistemas conceptuales exige un nivel de análisis superior al nivel individual. El objetivo primero en este caso no es comprender cómo los individuos aislados aprenden a utilizar metáforas conceptuales y otros sistemas conceptuales corporeizados, ni a estudiar las dificultades que podrían encontrar en su aprendizaje, la manera en que podrían perder su uso a consecuencia de lesiones cerebrales, o cualquier cosa de este tipo. Se trata de caracterizar, a través de centenas de expresiones verbales y de otras manifestaciones del acto de significar (como los gestos, por ejemplo), la estructura de las inferencias que se muestran en estas metáforas que son utilizadas por una comunidad de personas que operan con las mismas proyecciones.

Por ejemplo, cuando un hispanohablante entiende «el invierno quedó atrás», puede deducir implícitamente y sin esfuerzo que el otoño precedente no solamente quedó atrás, 
sino también que quedó todavia más atrás que el invierno. Igualmente, si lee «tenemos toda la vida por delante», comprenderá implícitamente que el próximo invierno está más cerca de él que, por ejemplo, su jubilación. El objeto de análisis de las ideas corporeizadas (ya sean matemáticas o no) se sitúa entonces en un nivel supra-individual, en el uso comunitario de, y las prácticas con, las proyecciones y las redes de inferencia metafóricas. Una gran comunidad de personas que emplea las mismas redes de metáforas y las mismas proyecciones en forma sistemática constituye una cultura. En lo que respecta a las ideas de lo cotidiano tales como el tiempo, estas culturas pueden corresponder de manera natural a grupos étnicos que hayan compartido un espacio geo-social durante siglos (como es el caso de los Aimara en los Andes). Pero puede existir una diversidad cultural en los modos de producción y sus prácticas, incluso de la matemática, independientemente de todo reagrupamiento étnico o geográfico. Este es el caso, por ejemplo, de ciertas escuelas de matemática que, no permitiendo el uso de la prueba por reducción al absurdo (como la escuela constructivista de Brouwer), excluyen explícitamente este método de sus prácticas. Se desprende de estas prácticas y rasgos culturales en matemática, una visión particular de qué es la matemática y de cuál es su naturaleza, las que tienen consecuencias matemáticas y filosóficas importantes.

\section{LA RELATIVIDAD DE LA VERDAD EN LOS CONCEPTOS IMAGINARIOS}

En lo concerniente a las entidades imaginarias, lo que se califica como «verdad» es relativo a las metáforas conceptuales subyacentes, es decir, a la organización de las inferencias en la red de proyecciones que lo sustenta. Por ejemplo, «el verano pasado», puede ser conceptualizado como situado detrás de nosotros en tanto que utilicemos la metáfora conceptual general, que determina una orientación particular del cuerpo frente a los acontecimientos concebidos metafóricamente en el tiempo - el futuro estando entonces situado ante nosotros y el pasado detrás de nosotros-. Como vimos, esta manera de conceptualizar el tiempo, aunque sea bastante común en el mundo, no es universal. Para un interlocutor Aimara procedente del altiplano andino, no es verdad que la expresión «el invierno está atrás» trata sobre un evento que ya ha acontecido. De hecho, esta frase significa para él exactamente lo contrario: ¡que el invierno en cuestión aún no acontece! Los Aimara se basan en una metáfora conceptual espaciotemporal diferente, de donde se desprende un conjunto diferente de «verdades». Como mencionamos anteriormente, el mismo fenómeno se produce en el dominio de la matemática con, por ejemplo, las diferentes ramas de la teoría contemporánea de conjuntos. Estas diferentes «teorías de conjuntos» producen verdades diversas caracterizadas por listas de axiomas diferentes. Vemos, por tanto, que no hay verdad definitiva y trascendental en materia de sistemas conceptuales surgidos de la imaginación humana. En nuestros ejemplos que tratan sobre el tiempo, no hay verdad definitiva en cuanto a la posición metafórica del futuro (ni del pasado). Igualmente ocurre con los conjuntos, de los que no puede decidirse definitivamente si tienen tal o cual propiedad, como la auto-pertenencia, por ejemplo. Toda verdad depende de detalles en la proyección de la metáfora conceptual subyacente. Esta idea es de una importancia primordial mientras se trate de conceptos matemáticos. La verdad que contienen no está oculta en la estructura del universo, sino que reposa más bien en los mecanismos del pensamiento humano y, más precisamente, en las proyecciones conceptuales corporeizadas subyacentes que han permitido crearlos. 


\section{Conclusión}

¿Cuál es la naturaleza de la matemática? ¿De dónde viene este cuerpo de conocimientos abstracto, eficaz, objetivo y tan bien organizado? Aquí defiendo la propuesta que emerge de lo imaginario, sostenido por la biología del cuerpo humano y por su mente, así como por el lenguaje y las prácticas culturales humanas. En particular, los mecanismos cognitivos ordinarios del imaginario humano — tales como la metáfora conceptual - forman parte integrante de la producción y de la creación de la propia matemática, jugando un papel constitutivo en la formalización matemática en tanto que tal, e incluso en la producción de los axiomas que crean nueva matemática. Las metáforas conceptuales (así como otros mecanismos como el «blending» conceptual y el movimiento ficticio, que no he tenido espacio aquí para tratar), en tanto que manifestaciones de mecanismos cognitivos muy eficaces y fluidos que tienen por objeto preservar las inferencias, juegan un papel fundamental en la creación de muchos conceptos matemáticos.

A partir del estudio de los gestos es posible ver que las metáforas conceptuales que sustentan las expresiones lingüísticas metafóricas en el campo del cálculo infinitesimal, por ejemplo, no son metáforas «muertas» (Núñez 2006; Marghetis y Núñez 2013). El análisis de la coproducción verbal-gestual de los matemáticos confirma la realidad psicológica y cognitiva de las metáforas conceptuales que sustentan, por ejemplo, las nociones de límites $\mathrm{y}$ de continuidad de funciones (y sus estructuras de inferencias). Al apoyarnos en estos estudios de gestos, podemos afirmar que estos matemáticos, además de utilizar las expresiones lingüísticas metafóricas, piensan en términos dinámicos —en tiempo real-. Es importante comprender que estos gestos no son simples instrumentos pedagógicos, ni tentativas para tornar las cosas abstractas más palpables. Estos gestos son de hecho manifestaciones vivas de la estructura de inferencias a partir de la cual se constituyen las ideas matemáticas fundamentales (tales como los límites o la continuidad), ideas constitutivas y dotadas de sentido, que no pueden ser tomadas por expresiones y definiciones puramente formales. Así, la matemática, que representa probablemente el más abstracto de los sistemas conceptuales, está, a fin de cuentas, anclada en nuestro cuerpo, nuestra mente, en nuestras prácticas lingüísticas y culturales y en el imaginario que sustentan.

\section{REFERENCIAS BIBLIOGRÁFICAS}

Barwise, Jon y Lawrence Moss. 1991. Hypersets. The Mathematical Intelligencer 13/4: 31-41.

Bates, Elizabeth y Fred Dick. 2002. Language, gesture, and the developing brain. Developmental Psychobiology 40: 293-310.

Cienki, Alan. 1998. Metaphoric gestures and some of their relations to verbal metaphoric expressions, in Jean-Pierre Koenig, ed., Discourse and Cognition, 189-204. Stanford, CA: CSLI Publications.

Courant, Robert y Herbert Robbins. 1978. What is mathematics? Oxford: Oxford University Press.

Cornejo, Carlos, Franco Simonetti, Agustín Ibañez, Nerea Aldunate, Francisco Ceric, Vladimir López, y Rafael Núñez. 2009. Gesture and metaphor comprehension: electrophysiological evidence of cross-modal coordination by audiovisual stimulation. Brain and Cognition 70/1: 42-52.

Fauconnier, Gilles y Mark Turner. 1998. Conceptual integration networks. Cognitive Science 22:133-187.

- 2002. The way we think: Conceptual blending and the mind's hidden complexities. New York: Basic Books.

Goldin-Meadow, Susan. 2003. Hearing gesture: How our hands help us think. Cambridge, MA: Belknap. 
Iverson, Jana y Esther Thelen. 1999. Hand, mouth, and brain: The dynamic emergence of speech and gesture, 19-40. In Rafael Núñez y Walter Freeman, eds, Reclaiming cognition: The primacy of action, intention, and emotion. Thorverton, UK: Imprint Academic.

Johnson, Mark. 1987. The Body in the mind: The Bodily basis of meaning, imagination, and reason. Chicago: University of Chicago Press.

Kendon, Adam. 2000. Language and gesture: Unity or duality? In David McNeill, ed., Language and gesture, 47-63. Cambridge, UK: Cambridge University Press.

Kita, Sotaro y James Essegbey. 2001. Pointing left in Ghana: How a taboo on the use of the left hand influences gestural practice. Gesture 1: 73-95.

Lakoff, George. 1993. The contemporary theory of metaphor. In Andrew Ortony, ed., Metaphor and thought (2nd ed.), 202-251. Cambridge: Cambridge University Press.

Lakoff, George y Mark Johnson. 1980. Metaphors we live by. Chicago: University of Chicago Press.

Lakoff, George y Rafael Núñez. 2000. Where mathematics comes from: How the embodied mind brings mathematics into being. New York: Basic Books, 2000.

Marghetis, Tyler y Rafael Núnez. 2013. The motion behind the symbols: a vital role for dynamism in the conceptualization of limits and continuity in expert mathematics. Topics in cognitive science 5/2: 299316.

McNeill, David. 1992. Hand and Mind: What gestures reveal about thought. Chicago: Chicago University Press.

Núñez, Rafael. 2006. Do real numbers really move ? Language, thought, and gesture: The embodied cognitive foundations of mathematics. In Reuben Hersh, ed., 18 unconventional essays on the nature of mathematics, 160-181. New York: Springer.

Núñez, Rafael y Kensy Cooperrider. 2013. The tangle of space and time in human cognition. Trends in Cognitive Sciences 17/5: 220-229.

Núñez, Rafael y Carlos Cornejo. 2012. Facing the sunrise: Cultural worldview underlying intrinsic-based encoding of absolute frames of reference in Aymara. Cognitive Science 36: 965-991.

Núñez, Rafael y George Lakoff. 1998. What did Weierstrass really define? The cognitive structure of natural and $\varepsilon-\delta$ continuity. Mathematical Cognition 4/2: 85-101.

$-\mathrm{y}-$. 2005. The cognitive foundations of mathematics: The role of conceptual metaphor. In Jamie Campbell, ed., Handbook of mathematical cognition, 109-124. New York: Psychology Press.

Núñez, Rafael y Eve Sweetser. 2006. With the Future Behind Them: Convergent evidence from language and gesture in the crosslinguistic comparison of spatial construals of time. Cognitive Science 30: 401-450.

Simmons, George F. 1985. Calculus with analytic geometry. New York: McGraw-Hill.

Sweetser, Eve. 1990. From Etymology to pragmatics: Metaphorical and cultural aspects of semantic structure. New York: Cambridge University Press.

Talmy, Leonard. 1996. Fictive motion in language and «ception». Language and space 21:1-276.

RAfael NúÑEz is professor of cognitive sciences at the University of California, San Diego, where he is the director of the Embodied Cognition Laboratory. He grew up in Chile, obtained his doctoral degree in Switzerland, and completed his post-doctoral work in Stanford University and University of California, Berkeley. He investigates cognition - especially conceptual systems and imagination- from the perspective of the embodied mind. His multidisciplinary approach uses methods such as psycholinguistic experiments, gesture studies, brain imaging, and field research with isolated indigenous groups. His book, Where Mathematics Comes From: How the Embodied Mind Brings Mathematics into Being (with George Lakoff) presents a novel theoretical framework for understanding the human nature of mathematics and its foundations. He is the founding co-director of the Fields Cognitive Science Network for empirical study of the nature of mathematics, based at the Fields Institute in Toronto.

Adress: Department of Cognitive Sciences. University of California, San Diego. 9500 Gilman Drive. La Jolla, CA 92093. U.S.A. Email: rnunez@ucsd.edu 